\title{
The global effect of aripiprazole monotherapy in the spectrum of symptoms in bipolar disorder: a meta-analysis
}

\author{
Konstantinos N Fountoulakis
}

From $1^{\text {st }}$ International Congress on Neurobiology and Clinical Psychopharmacology and European

Psychiatric Association Conference on Treatment Guidance

Thessaloniki, Greece. 19-22 November 2009

\section{Background}

The current article is a systematic registration and meta-analysis of the available clinical trials concerning the usefulness of aripiprazole in the treatment of bipolar disorder in affective as well as in psychotic symptoms.

\section{Materials and methods}

A systematic MEDLINE and repositories search concerning treatment guidelines and clinical trials for aripiprazole in bipolar disorder.

\section{Results}

The pooled effect size for acute manic symptoms was equal to 0.34 . The NNT was 6 for aripiprazole vs placebo concerning response at week 3 and equal to 14 concerning remission. The average day response started was day 3 . The suicide rates were negligible for all groups. In contrast to the individual isolated trials, the meta-analysis for acute bipolar depression suggests a significant difference at week 8 with an effect size 0.17 . The meta-analysis of the 4 trials which reported the efficacy of aripiprazole on psychotic symptoms during an acute manic/mixed episodes suggests that the effect size vs. placebo was equal to 0.14 but a more reliable and accurate estimation is 0.18 for the total PANSS score. The effect was higher for the PANSS positive subscale (0.28), PANSS hostility subscale $(0.24)$ and PANSS cognitive subscale $(0.20)$, and lower for the PANSS negative (0.12). No data on the psychotic symptoms during the depressive phase of bipolar illness exist. The maintenance data suggest that the median survival time for the emergence of a manic episode for the aripiprazole group was not evaluable, while the median survival time for placebo was 118-203 days depending on the clinical subpopulation. Concerning psychotic symptoms, at week 26 all except the total PANSS score showed a significant superiority of aripiprazole over placebo $(\mathrm{d}=0.28$ for positive, $d=0.38$ for the cognitive and $d=0.71$ for the hostility subscales) and at week 100 the results were similar $(\mathrm{d}=0.42,0.63$ and 0.48 respectively).

\section{Conclusion}

The data analysed for the current study support the usefulness of aripiprazole during all phases of bipolar illness, inspite of the rather weak effect on depression and that the efficacy during the maintenance period is proven against new manic episodes and in patients with an index manic episode who responded to aripiprazole during the acute phase.

Published: 22 April 2010

3rd Department of Psychiatry, Aristotle University of Thessaloniki, Greece

doi:10.1186/1744-859X-9-S1-S59

Cite this article as: Fountoulakis: The global effect of aripiprazole monotherapy in the spectrum of symptoms in bipolar disorder: a metaanalysis. Annals of General Psychiatry 2010 9(Suppl 1):S59. 